\title{
Mechanisms of Postural Tachycardia Syndrome
}

Key words: autonomic dysfunction, vascular tone, blood pressure, heart rate

The postural orthostatic tachycardia syndrome (POTS) (1), or postural tachycardia syndrome (2), is characterized by a persistent tachycardia while upright, which is associated with palpitation, disabling fatigue, near syncope, exercise intolerance, diaphoresis and lightheadedness or dizziness. This clinical entity was named Postural Orthostatic Tachycardia Syndrome by Schondorf and Low in 1993 (1), and then simply Postural Tachycardia Syndrome by Low and associates in 1995 (2). This specific syndrome was already described in 4 patients by MacLean et al in 1944 (3). Their patients presented with orthostatic tachycardia along with a mild fall in blood pressure, and complained of palpitation, lightheadedness, weakness and exercise intolerance. MacLean and associates attributed properly this clinical manifestation to the defect in venous return to the heart.

Several possible mechanisms have been proposed for the underlying pathogenesis of POTS (4). They include hypovolemia, mild or attenuated form of autonomic dysfunction, and hyperdynamic beta-adrenergic function. Dysfunction in the central autonomic network is another possible mechanism (5). In some patients, the symptoms followed viral infection (1), and immune-mediated pathogenesis could be involved, at least in part, in this syndrome. Streeten and Scullard extensively investigated the responses of arterioles and veins to norepinephrine infusion in patients with different types of orthostatic intolerance (6). Patients with POTS, or hyperadrenergic orthostatic tachycardia, showed normal arteriolar response, i.e., rise in diastolic pressure. In contrast, constrictive responses of the foot vein to norepinephrine was exaggerated, i.e., supersensitive response of the postganglionically denervated lower limb veins (6). Via this abnormal function of the lower limb veins, there could be excessive gravitational pooling of blood in the leg vein, a common finding among patients with different types of orthostatic intolerance.

Patients with diffuse autonomic insufficiency showed supersensitive response of diastolic blood pressure and of venous constriction to norepinephrine infusion (6). This suggests that arteriolar and venous innervation is impaired in patients with diffuse autonomic insufficiency. In contrast, patients with hyperadrenergic orthostatic tachycardia or hyperadrenergic orthostatic hypotension have only impaired venous constrictive response. Hypovolemia could be attributed to a different clinical manifestation from hyperadrenergic orthostatic tachy- cardia and hypotension; mild or no hypovolemia in the former and more severe hypovolemia in the latter (6).

Grubb and associates (7) showed an exaggerated response of heart rate to a low dose of isoproterenol in patients with POTS. Therefore, patients with POTS have a deficiency in peripheral vascular function, especially in the vein, due to a mild form of autonomic dysfunction, and while standing an excessive compensatory tachycardia might emerge through an intact or supersensitive beta-adrenergic system of the heart.

In this issue of the Journal, Kishi et al (8) describe a young woman who presented with palpitation, low-grade fever, weight loss and hyperhydrosis.

\section{See also $\mathrm{p} 1032$.}

Head-up tilt test showed orthostatic tachycardia with an increase of heart rate by $40 / \mathrm{min}$ but without any appreciable changes in arterial blood pressure. To determine the possible mechanism of orthostatic tachycardia in this woman, several types of autonomic function tests were undertaken. Heart rate variability and the Valsalva ratio were within physiologic ranges suggesting an intact cardiac autonomic function. Plasma norepinephrine level was normally elevated while standing, but blood pressure response to norepinephrine infusion was almost totally abolished. These data indicate that alpha-adrenergic response of arterioles was impaired in this patient. This impairment was also suggested by a marked decrease in blood pressure and pulse pressure in phase II of the Valsalva maneuver. In phase II of the Valsalva maneuver, cardiac output decreases via a reduction in venous return, leading to an increase in reflex sympathetic tone. This increase in sympathetic tone increases peripheral vascular resistance and thereby elevates both systolic and diastolic blood pressures. This compensatory mechanism was not seen in the patient.

Although Streeten and Scullard stressed that the disordered venous innervation exists exclusively in the lower limbs in patients with hyperadrenergic orthostatic hypotension $(6,9)$, the present case could have generalized adrenergic denervation because the decreased sweat response to iontophoresis of acetylcholine was seen in both upper limbs and lower limbs. Another characteristic finding of this patient was an excessive blood pressure elevation in the phase IV of the Valsalva maneuver. In this specific phase of the maneuver, the increase in venous return superimposed on the presence of the increased peripheral vascular resistance which continued from phases II and III could lead to increases in both systolic and diastolic blood pressures and in pulse pressure. The mechanism of this exaggerated elevation of blood pressure in phase IV was not 
investigated in this patient. Grubb et al showed a supersensitive response of the heart rate to isoproterenol in patients with POTS (7). The present authors suggested a hyperadrenergic state as the underlying mechanism of the exaggerated blood pressure response. Since elevation of the plasma norepinephrine was within the physiologic range, a supersensitive betaadrenergic system could be present in the heart.

The underlying mechanisms of POTS could be multifactorial $(1,4,5)$. It seems likely that a mild form of autonomic dysfunction (4) could be involved in the pathogenesis of the patient of Kishi et al (8). It is, however, still unclear whether partial autonomic dysfunction is functional or anatomic. It should be noted that full blown clinical manifestation of pure autonomic failure might ensue in approximately $10 \%$ of the patients (1), although the symptoms of the patient of Kishi et al were improved with an adequate treatment (8).

Hiroshi INOUE, MD

The Second Department of Internal Medicine, Toyama Medical and Pharmaceutical University, 2630 Sugitani, Toyama 930-0194

\section{References}

1) Schondorf R, Low PA. Idiopathic postural orthostatic tachycardia syndrome: An attenuated form of acute pandysautonomia? Neurology 43 : $132-137,1993$.

2) Low PA, Opfer-Gehrking TL, Textor S, et al. Postural tachycardia syndrome. Neurology 45: S18-S25, 1995.

3) MacLean AR, Allen EV, Magath TB. Orthostatic tachycardia and orthostatic hypotension: Defects in the return of venous blood to the heart. Am Heart J 27: 145-163, 1944.

4) Jacob G, Shannon JR, Costa F, et al. Abnormal norepinephrine clearance and adrenergic receptor sensitivity in idiopathic orthostatic intolerance. Circulation 99: 1706-1712, 1999.

5) Benarroch EE. The central autonomic network: Functional organization, dysfunction, and perspective. Mayo Clin Proc 68: 988-1001, 1993.

6) Streeten DH, Scullard TF. Excessive gravitational blood pooling caused by impaired venous tone is the predominant non-cardiac mechanism of orthostatic intolerance. Clin Sci 90: 277-285, 1996.

7) Grubb BP, Kosinski DJ, Boehm K, Kip K. The postural orthostatic tachycardia syndrome: A neurocardiogenic variant identified during head-up tilt table testing. PACE 20: 2205-2212, 1997.

8) Kishi Y, Sasaki H, Kondo T, et al. Postural tachycardia syndrome in a 28year-old Japanese woman. Intern Med 40: 1032-1036, 2001.

9) Streeten DH. Pathogenesis of hyperadrenergic orthostatic hypotension: Evidence of disordered venous innervation exclusively in the lower limbs. J Clin Invest 86: 1582-1588, 1990. 